\title{
Longitudinal progression of clinical variables associated with graded liver injury in COVID-19 patients
}

\author{
Justin Y. Lu ${ }^{1} \cdot$ Harnadar Anand ${ }^{1} \cdot$ Shalom Z. Frager ${ }^{2} \cdot$ Wei Hou $^{3} \cdot$ Tim Q. Duong $^{1}$ (I)
}

Received: 20 March 2021 / Accepted: 18 June 2021 / Published online: 15 July 2021

(c) Asian Pacific Association for the Study of the Liver 2021

\begin{abstract}
Background Hospital-acquired liver injury is associated with worse outcomes in COVID-19. This study investigated the temporal progression of clinical variables of in-hospital liver injury in COVID-19 patients.

Methods COVID-19 patients $(n=1361)$ were divided into no, mild and severe liver injury (nLI, mLI and sLI) groups. Time courses of laboratory variables were time-locked to liver-injury onset defined by alanine aminotransferase level. Predictors of liver injury were identified using logistic regression.

Results The prevalence of $\mathrm{mLI}$ was $39.4 \%$ and sLI was 9.2\%. Patients with escalated care had higher prevalence of sLI (23.2\% vs. $5.0 \%, p<0.05$ ). sLI developed 9.4 days after hospitalization. sLI group used more invasive ventilation, anticoagulants, steroids, and dialysis $(p<0.05)$. sLI, but not $\mathrm{mLI}$, had higher adjusted mortality odds ratio $(=1.37$ [95\% CI $1.10,1.70$ ], $p=0.005$ ). Time courses of the clinical variables of the sLI group differed from those of the nLI and mLI group. In the sLI group, alanine aminotransferase, procalcitonin, ferritin, and lactate dehydrogenase showed similar temporal profiles, whereas white-blood-cell count, D-dimer, C-reactive protein, respiration and heart rate were elevated early on, and lymphocyte and $\mathrm{SpO} 2$ were lower early on. The top predictors of sLI were alanine aminotransferase, lactate dehydrogenase, respiration rate, ferritin, and lymphocyte, yielding an AUC of $0.98,0.92,0.88$ and 0.84 at $0,-1,-2$ and -3 days prior to onset, respectively. Conclusions This study identified key clinical variables predictive of liver injury in COVID-19, which may prove useful for management of liver injury. Late onset of sLI and more aggressive care are suggestive of treatment-related hepatotoxicity.
\end{abstract}

Keywords SARS-CoV-2 $\cdot$ Liver failure $\cdot$ Liver dysfunction $\cdot$ Acute kidney injury $\cdot$ Aminotransferases $\cdot$ Aspartate aminotransferase $\cdot$ Multi-organ failure $\cdot$ Cytokine storm

\section{Introduction}

Coronavirus disease 2019 (COVID-19) [1,2] caused by the novel severe acute respiratory syndrome coronavirus 2 (SARS-CoV-2) has evolved into a global pandemic with devastating morbidity and mortality. The widespread outbreaks and the likelihood of resurgences have strained

Tim Q. Duong

Tim.duong@einsteinmed.org

1 Department of Radiology, Albert Einstein College of Medicine and Montefiore Medical Center, 111 E 210th St, Bronx, NY 10467, USA

2 Department of Medicine, Division of Liver Transplant, Albert Einstein College of Medicine and Montefiore Medical Center, Bronx, NY, USA

3 Department of Family, Population and Preventive Medicine, Stony Brook Medicine, Stony Brook, NY, USA and will likely continue to strain healthcare resources for extended period. SARS-CoV-2 infection affects many vital organs and, in severe cases, could result in multiple organ failure. Among many organs affected, in-hospital acquired liver injury has been reported to be associated with worse COVID-19 outcomes [3-10]. The effects of COVID-19 on the liver remain incompletely understood [3-10]. The liver could be damaged by direct viral infection, indirect host-immune responses (i.e., thrombosis, cytotropic and cytokine-mediated immune responses, amongst others) and/ or hepatotoxicity from COVID-19 therapies that include antiviral, antibacterial, and immuno-modulatory drugs, amongst others [11-14].

The temporal progression of in-hospital liver injury and its relations with clinical variables and clinical outcomes are unknown. To our knowledge, there has been no systematic evaluation of the temporal profiles of clinical variables leading up liver injury in COVID-19, and how these temporal 
profiles can predict liver injury and thus inform clinical decision making. Improved understanding of in-hospital liver injury associated with COVID-19 could lead to better patient management that include more frequent monitoring of liver function tests, particularly in patients treated with Remdesivir $^{\circledR}$ and patients with advanced liver disease [14].

The goal of this study was to investigate the temporal progression of clinical variables associated with in-hospital liver injury in COVID-19 patients. We analyzed the temporal profiles of different clinical variables with time-lock to liver injury onset defined by alanine aminotransferase level. We grouped patients into graded (no, mild and severe) liver injury. The effects of treatments (such as invasive mechanical ventilation, anticoagulants, and steroids) were also analyzed with respect to graded liver injury.

\section{Methods}

\section{Study population and data collection}

This retrospective single-center study was approved by the Stony Brook Institutional Research Board with an exemption for informed consent and a HIPAA waiver. The study followed the Strengthening the Reporting of Observational Studies in Epidemiology (STROBE) reporting guidelines for cohort studies [15], except sample size and sensitivity analyses were not calculated because no a priori data exist for longitudinal liver injury in COVID-19.

Our COVID-19 Persons Under Investigation Registry consisted of 6678 patients clinically suspected of COVID-19 infection from February 7, 2020 to June 30, 2020 [16-20], of which 2892 were tested positive using real-time polymerase chain reaction test for severe acute respiratory syndrome coronavirus 2 (SARS-CoV-2) on a nasopharyngeal swab specimen. Exclusion criteria included patients who were not hospitalized, without alanine aminotransferase (ALT) and aspartate aminotransferase (AST) data, less than 18 years old, and still in the hospital. There were 1361 hospitalized COVID-19 patients meeting the inclusion/exclusion criteria.

Patients were then divided into three groups based on liver injury (LI) defined by ALT level [21]: (1) no liver injury (nLI) with ALT $<1$ ULN $[<40 \mathrm{U} / \mathrm{L}]$ and AST $<1$ ULN $[<35 \mathrm{U} / \mathrm{L}]$, (2) mild liver injury $(\mathrm{mLI})$ with ALT $=1-5$ ULN and AST $=1-3$ ULN, and (3) severe livery injury (sLI) with ALT $\geq 5$ ULN and AST $\geq 3$ ULN. In the general floor cohort, there were $605 \mathrm{nLI}, 389 \mathrm{mLI}$, and 52 sLI patients. In the ICU cohort, there were $95 \mathrm{nLI}, 147 \mathrm{mLI}$, and $315 \mathrm{sLI}$ patients.

Although AST, ALT, ALP and total bilirubin are commonly used tests to assess liver injury and are associated with increased mortality risk in COVID-19 [22], we chose to time lock to ALT because ALT is more liver specific than the other liver function biomarkers [23-25] (see Discussion).

Demographics and chronic comorbidities, longitudinal vital signs, laboratory blood tests, and blood gases were extracted from electronic medical records. Demographic data included age, gender, ethnicity, and race. Chronic comorbidities included smoking, diabetes, hypertension, asthma, chronic obstructive pulmonary disease (COPD), coronary artery disease, heart failure, cancer, immunosuppression, and chronic kidney disease. Longitudinal laboratory tests included creatinine ( $\mathrm{Cr}$ ), procalcitonin (PROCAL), aspartate transaminase (AST), alanine aminotransferase (ALT), ferritin (FERR), lactate dehydrogenase (LDH), white blood cell count (WBC), C-reactive protein (CRP), lymphocytes count (LYMPH), D-dimer (DDIM), brain natriuretic peptide (BNP), alkaline phosphatase (ALP), total bilirubin, prothrombin time, total protein, and albumin. Longitudinal vital signs and blood gas variables included heart rate (HR), respiratory rate (RR), pulse oxygen saturation (SpO2), temperature (TEMP), and $\mathrm{pH}$. FiO2 was administered and well documented in ICU patients but not usually so in general floor patients, and thus $\mathrm{FiO} 2$ was not tabulated.

Laboratory test variables were plotted across time with time lock $(t=0)$ to LI onset (defined by elevated ALT), along with data 3 days before and 3 days after LI onset. For comparison, time series data for no LI patients were time locked $(t=0)$ to 3 days after ED admission, along with data 3 days before and 3 days after that time point. For ease of comparison, individual laboratory test values were normalized to the no LI group at $t=0$ for individual patients.

\section{Predictive models}

Logistic regression models were used to rank the importance of clinical variables leading to liver injury at each day prior to onset. Ranking was performed using all clinical variables except BNP, ALP, total bilirubin, total protein, and prothrombin time due to significant missing data at multiple time points. Prediction performance was evaluated for individual top variables and the combined top variables in predicting both sLI and $\mathrm{mLI}$ as well as sLI only at different days prior to onset. For the combined prediction models, AUC calculation excluded AST due to its strong correlation with ALT. Prediction performance was evaluated using the area under the curve (AUC) of the receiver operating characteristic (ROC) curve.

\section{Statistical analysis}

All statistical analyses were performed using Python packages (Sklearn and Statsmodels) and Statistical Analysis System (SAS) software (Cary, NC, USA). Frequencies and percentages for categorical variables between $\mathrm{nLI}$ and mild 
LI and sLI using $\chi^{2}$ or Fisher's exact tests. Age, expressed as median (IQR), was compared between groups using ANOVA and post hoc pairwise $t$-test. Mortality rates were compared between groups with logistic regression adjusted with covariates (age, gender, ethnicity, and significant comorbidities). Differences between nLI, mLI, and sLI, for clinical variables in time series graphs were analyzed via linear mixed models and least-squares means. $p$-values $<0.05$ were considered statistically significant and corrected using the Bonferroni method for appropriate, multiple tests.

\section{Results}

Figure 1 shows the patient selection flowchart. Of the 1361 hospitalized COVID-19 patients who met the inclusion and exclusion criteria, (1) 1046 patients were admitted to general floor and never transferred to ICU, (2) 214 patients upgraded to ICU from general floor, and (3) 101 patients directly admitted to ICU from emergency room. In the never-upgrade general floor group $(n=1046), 57.8 \%$ had nLI, $37.2 \%$ had $\mathrm{mLI}$, and $5.0 \%$ had sLI. In the direct ICU plus, the upgraded ICU group ( $n=315), 30.1 \%$ had $\mathrm{nLI}, 46.7 \%$ had $\mathrm{mLI}$, and $23.2 \%$ had sLI.

Table 1A summarizes patient demographics, comorbidities and select treatments categorized by $\mathrm{nLI}, \mathrm{mLI}$, and sLI. Of all hospitalized COVID-19 patients, $51.43 \%$ of patients had $\mathrm{nLI}, 39.38 \%$ had $\mathrm{mLI}$, and $9.18 \%$ had sLI. nLI patients were older than mLI patients $(p<0.05)$, followed by sLI patients $(p<0.05)$. Sex $(p<0.05)$ and ethnicity $(p<0.05)$ were significantly different amongst groups. Race was significantly different between nLI and mLI $(p<0.05)$ and between nLI and sLI $(p<0.05)$. Smoking, diabetes, hypertension, COPD, coronary artery disease, cancer, chronic kidney disease $(p<0.05)$, but not asthma

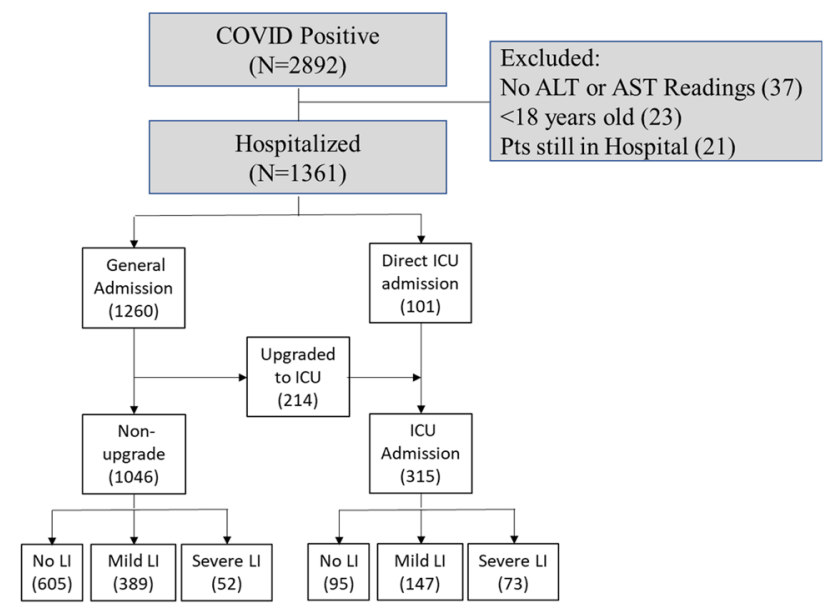

Fig. 1 Flowchart of patient selection. Pts patients, ICU intensive care unit, $A L T$ alanine aminotransferase, $A S T$ aspartate aminotransferase and immunosuppression ( $p>0.05$ ), were significantly different between $\mathrm{nLI}$ and mLI. Smoking, coronary artery disease, heart failure and cancer $(p<0.05)$, but not diabetes, hypertension, asthma, COPD immunosuppression and chronic kidney disease $(p>0.05)$, were significantly different between nLI and sLI. There was no significant difference in comorbidities between mLI and sLI patients. Overall, nLI patients generally had more comorbidities than $\mathrm{mLI}$ and sLI patients. By comparison, in the ICU group, $30.1 \%$ of patients had nLI, $46.7 \%$ had $\mathrm{mLI}$, and $23.2 \%$ had sLI.

Of all hospitalized COVID-19 patients, the unadjusted mortality rates for $\mathrm{nLI}, \mathrm{mLI}$, and sLI were, respectively, $14.3 \%, 12.3 \%$ and $36.0 \%$. The adjusted mortality OR for mLI was 1.16 [95\% CI 0.81, 1.68] ( $p=0.415)$, not significantly different from nLI. The adjusted mortality OR for sLI was 1.37 [95\% CI 1.10, 1.70] $(p=0.005)$, significantly different from nLI. Adjustment was made for age, gender, hypertension, and diabetes.

sLI patients received markedly more invasive and noninvasive mechanical ventilation, prophylactic and therapeutic anticoagulants, steroids, and dialysis than both $\mathrm{mLI}$ and nLI patients (Table $1 \mathrm{~B}, p<0.05$ ). mLI patients received significantly more invasive mechanical ventilation, prophylactic anticoagulants, and steroids than nLI patients $(p<0.05)$.

The histograms of the days of hospitalization before patients develop liver injury are plotted in Fig. 2. mLI developed an average of 2.6 days after hospital admission and $43.9 \%$ of the mLI patients had liver injury on the day of hospital admission. sLI developed an average of 9.4 days after hospital admission, and $16.0 \%$ of patients had sLI on the day of admission.

Figure 3 depicts the time series of laboratory tests relative to liver injury onset for nLI, mLI, and sLI cohorts. For ease of comparison, these laboratory values were normalized to the nLI group at $t=0$ by individual patients. The ALT of nLI group remained low and time invariant, whereas ALT of the mLI group was $3 \times$ and the ALT of sLI group was $15 \times$ that of $\mathrm{nLI}$ at $t=0$. An apparent observation is that the time courses of sLI were markedly different from those of $\mathrm{mLI}$ and nLI, whereas those of mLI and nLI were similar.

For sLI, AST, ALP, and total bilirubin (liver enzymes) showed similar time courses. Procalcitonin, ferritin, LDH also showed similar time courses as ALT. Bilirubin, procalcitonin, and ferritin remained elevated or further increased days after sLI onset.

For the sLI, WBC, D-dimer, CRP, HR, RR were elevated throughout, whereas lymphocyte, $\mathrm{SpO} 2$ and prothrombin time were lower throughout. $\mathrm{Cr}$, total protein and albumin appeared to peak earlier relative ALT. BNP, temperature and $\mathrm{pH}$ were mostly similar amongst groups, although there were some differences at some time points. A drop in $\mathrm{pH}$ was seen on the day of the sLI onset. 
Table 1 (A) Demographic characteristics, comorbidities and (B) treatments, ICU admission, length of state (LOS), and unadjusted mortality rate of no liver injury $(\mathrm{nLI})$, mild liver injury $(\mathrm{mLI})$ and severe liver injury (sLI) patients

\begin{tabular}{|c|c|c|c|c|}
\hline & \multicolumn{4}{|c|}{ Patients, No. (\%) } \\
\hline & $\mathrm{nLI}(n=700)$ & $\mathrm{mLI}(n=536)$ & $\operatorname{sLI}(n=125)$ & $p$ value \\
\hline \multicolumn{5}{|l|}{ (A) } \\
\hline \multicolumn{5}{|l|}{ Demographics } \\
\hline Age, mean (sd) & $66.9(17.2)^{\mathrm{a}}$ & $58.4(16.0)^{\mathrm{b}}$ & $56.7(17.5)^{\mathrm{b}}$ & $<0.001$ \\
\hline Sex & & & & $<0.001$ \\
\hline Male & $332(47.4 \%)^{\mathrm{a}}$ & $356(66.4 \%)^{\mathrm{b}}$ & $96(76.8 \%)^{\mathrm{b}}$ & \\
\hline Female & $368(52.6 \%)$ & $180(33.6 \%)$ & $29(23.2 \%)$ & \\
\hline Ethnicity & & & & $<0.001$ \\
\hline Hispanic/Latino & $21.0 \%^{\mathrm{a}}$ & $32.6 \%^{\mathrm{b}}$ & $21.6 \%^{\mathrm{b}}$ & \\
\hline Non-Hispanic/Latino & $69.0 \%$ & $51.9 \%$ & $56.8 \%$ & \\
\hline Unknown & $10.0 \%$ & $15.5 \%$ & $21.6 \%$ & \\
\hline Race & & & & $<0.001$ \\
\hline Caucasian & $62.2 \%$ & $45.2 \%$ & $46.4 \%$ & \\
\hline African American & $8.9 \%$ & $5.8 \%$ & $4.0 \%$ & \\
\hline Asian & $2.1 \%$ & $5.0 \%$ & $5.6 \%$ & \\
\hline Unknown & $26.1 \%$ & $42.7 \%$ & $43.2 \%$ & \\
\hline \multicolumn{5}{|l|}{ Comorbidities } \\
\hline Smoking history & & & & 0.171 \\
\hline Current smoker & $5.0 \%$ & $3.9 \%$ & $4.8 \%$ & \\
\hline Former smoker & $24.6 \%$ & $18.7 \%$ & $19.2 \%$ & \\
\hline Never smoked & $63.3 \%$ & $72.2 \%$ & $71.2 \%$ & \\
\hline Unknown & $7.1 \%$ & $5.2 \%$ & $4.8 \%$ & \\
\hline Diabetes & $31.0 \%^{\mathrm{a}}$ & $23.0 \%^{\mathrm{b}}$ & $24.0 \%^{\mathrm{b}}$ & 0.005 \\
\hline Hypertension & $54.3 \%^{\mathrm{a}}$ & $45.2 \%^{\mathrm{b}}$ & $49.6 \%^{\mathrm{b}}$ & 0.005 \\
\hline Asthma & $5.3 \%$ & $7.3 \%$ & $7.2 \%$ & 0.376 \\
\hline COPD & $11.1 \%^{\mathrm{a}}$ & $6.4 \%^{\mathrm{b}}$ & $8.0 \%^{\mathrm{ab}}$ & 0.009 \\
\hline CAD & $18.0 \%^{\mathrm{a}}$ & $13.6 \%^{\mathrm{a}}$ & $9.6 \%{ }^{\mathrm{a}}$ & 0.015 \\
\hline Heart failure & $12.6 \%^{\mathrm{a}}$ & $5.1 \%^{\mathrm{b}}$ & $4.0 \% \mathrm{bc}$ & $<0.001$ \\
\hline Cancer & $13.3 \%^{\mathrm{a}}$ & $6.7 \%^{\mathrm{b}}$ & $5.6 \%^{\mathrm{ab}}$ & $<0.001$ \\
\hline Immunosuppression & $10.1 \%^{\mathrm{a}}$ & $5.8 \%^{\mathrm{b}}$ & $5.6 \%^{\mathrm{ab}}$ & 0.005 \\
\hline CKD & $13.7 \%^{\mathrm{a}}$ & $6.4 \%^{\mathrm{b}}$ & $10.4 \%$ ab & $<0.001$ \\
\hline \# of comorbidities per patient & & & & 0.155 \\
\hline 0 & $26.3 \%$ & $38.3 \%$ & $37.6 \%$ & \\
\hline 1 & $24.3 \%$ & $25.9 \%$ & $29.6 \%$ & \\
\hline 2 & $22.6 \%$ & $21.3 \%$ & $17.6 \%$ & \\
\hline 3 & $14.1 \%$ & $10.0 \%$ & $7.2 \%$ & \\
\hline 4 & $7.6 \%$ & $3.7 \%$ & $4.0 \%$ & \\
\hline 5 & $3.7 \%$ & $0.4 \%$ & $3.2 \%$ & \\
\hline 6 & $1.3 \%$ & $0.4 \%$ & $0.8 \%$ & \\
\hline 7 & $0.1 \%$ & 0 & 0 & \\
\hline \multicolumn{5}{|l|}{ (B) Treatments } \\
\hline IMV & $8.1 \%^{\mathrm{a}}$ & $22.3 \%^{\mathrm{b}}$ & $52.0 \%^{\mathrm{c}}$ & $<0.001$ \\
\hline Noninvasive MV & $6.9 \%^{\mathrm{a}}$ & $9.9 \%^{\mathrm{b}}$ & $22.4 \%^{\mathrm{c}}$ & $<0.001$ \\
\hline \multicolumn{5}{|l|}{ Anticoagulants } \\
\hline Prophylactic & $35.6 \%^{\mathrm{a}}$ & $44.0 \%^{\mathrm{b}}$ & $58.4 \%^{\mathrm{c}}$ & $<0.001$ \\
\hline Therapeutic & $16.3 \%^{\mathrm{a}}$ & $15.3 \%^{\mathrm{a}}$ & $33.6 \%^{\mathrm{b}}$ & $<0.001$ \\
\hline Steroids & $13.3 \%^{\mathrm{a}}$ & $26.1 \%^{\mathrm{b}}$ & $52.0 \%^{\mathrm{c}}$ & $<0.001$ \\
\hline Dialysis & $5.4 \%^{\mathrm{a}}$ & $4.7 \%^{\mathrm{b}}$ & $16.0 \%^{\mathrm{c}}$ & $<0.001$ \\
\hline$\%$ in ICU admission & $13.57 \%^{\mathrm{a}}$ & $27.43 \%^{\mathrm{b}}$ & $58.40 \%^{\mathrm{c}}$ & $<0.001$ \\
\hline Length of stay & $7.9(7.8)^{\mathrm{a}}$ & $12.8(13.6)^{\mathrm{b}}$ & $20.2(16.6)^{\mathrm{c}}$ & $<0.001$ \\
\hline Mortality (unadjusted) & $14.3 \%^{\mathrm{a}}$ & $12.3 \%^{\mathrm{a}}$ & $36.0 \%^{\mathrm{b}}$ & $<0.001$ \\
\hline
\end{tabular}

Group comparison of categorical variables in frequencies and percentages used $\chi^{2}$ test or Fisher exact tests. Group comparison of age in mean and standard deviations used the ANOVA. $p$ values are based on overall Chi-square tests. a $\mathrm{b} \mathrm{c}$ denote significant groups based on pairwise tests with Bonferroni correction for $p$ values

$C O P D$ chronic obstructive pulmonary disease, $C A D$ coronary artery disease, $C K D$ chronic kidney disease 
(A)

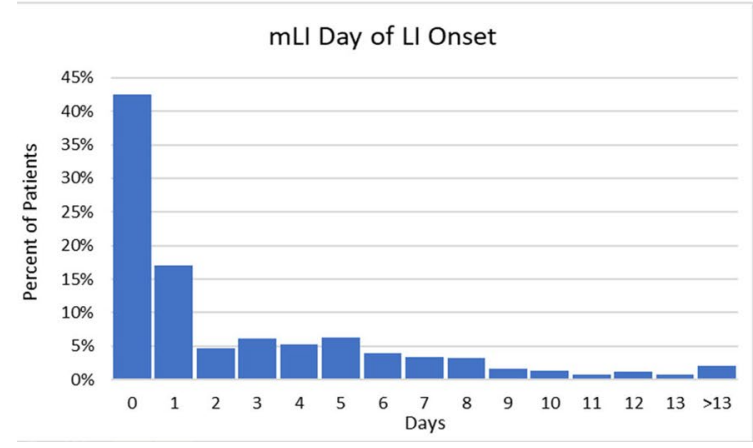

(B)

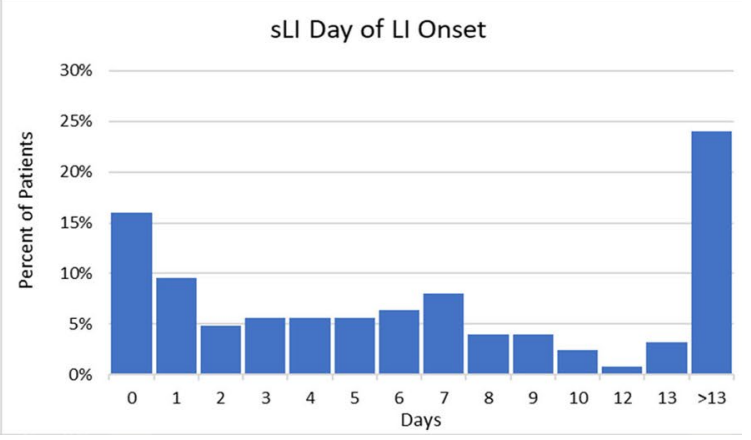

Fig. 2 Histogram of patients who developed $\mathbf{a}$ mild and $\mathbf{b}$ severe liver injury after the number of days in hospital. Time $=0$ represents admission date of patients. Average date until liver injury development for mLI patients was 2.6 days and sLI patients was 9.4 days

Table 2 shows the prediction performance (AUCs) of individual top variables and the combined top variables for both sLI and mLI as well as sLI only at different days prior to onset. AUCs were high on the day of onset and decreased -2 and -3 days before. For prediction of both SLI and $\mathrm{mLI}$, the top predictors were ALT, AST, LDH, lymphocyte, and RR; the AUCs of the predictive model combining these predictors were $0.81,0.79,0.78$ and 0.68 at $0,-1,-2$ and -3 days prior, respectively. For prediction of sLI only, the top predictors were ALT, AST, LDH, RR, ferritin, and lymphocyte; the AUCs of the predictive model combining these predictors were $0.98,0.92,0.88$ and 0.84 at $0,-1,-2$ and -3 days prior, respectively. Prediction of sLI was overall better than that of sLI and $\mathrm{mLI}$.

\section{Discussion}

This study investigated the temporal progression of clinical variables associated with graded hospital-acquired liver injury in COVID-19 patients. The major findings are: (1) sLI COVID-19 patients have higher prevalence of younger age, more male sex, invasion ventilation, anticoagulants, steroids and dialysis, (2) sLI, but not mLI, has significantly higher adjusted mortality than nLI, (3) COVID-19 patients develop sLI 9.4 days after hospitalization on an average, (4) the clinical variables time courses of the sLI group are markedly different from those of the $\mathrm{nLI}$ and $\mathrm{mLI}$ group, (5) for the sLI, ALT and a few liver panel laboratory variables, as well as procalcitonin, ferritin, and lactate dehydrogenase show similar temporal trajectories, whereas white-blood cell count, D-dimer, C-reactive protein, heart rate, respiration rate are elevated from the onset, and lymphocyte, $\mathrm{SpO} 2$ and prothrombin time are lower from the onset, and (6) the top predictors of sLI were ALT, AST, LDH, RR, ferritin and lymphocyte, and the AUCs of the predictive model combining these predictors were $0.98,0.92,0.88$ and 0.84 at $0,-1,-2$ and -3 days prior to liver injury onset, respectively.

\section{Liver injury confers a high mortality rate}

The sLI group received more invasive mechanical ventilation, anticoagulant, steroid, and dialysis, more likely to be admitted to ICU, stayed longer in the hospital, and had a higher adjusted mortality odds ratio, despite being younger and having fewer comorbidities, suggesting that SLI is an independent risk factor of worse COVID-19 outcomes. Mortality ORs varied and have been reported to be 1.48 [95\% CI 1.12-1.96] for elevated ALT [4], 1.03 [95\% CI 1.01-1.05] for elevated AST (another marker liver injury) [26], and 3.56 for ALT $>120 \mathrm{U} / \mathrm{L}$ [22]. These different findings are not unexpected and may depend on the timing of COVID-19 pandemic, patient cohorts, patient management by different hospitals, and definitions of liver injury, amongst others.

\section{Clinical variables time courses offer novel insight into COVID-19-related liver injury}

In contrast to previous studies [3-6] which typically assessed clinical variables at admission, our study analyzed graded liver injury and their temporal dynamics with respect to other clinical variables. Another novelty of this study is that we time-locked these longitudinal clinical variables to liver injury onset. If only clinical variables at hospital admission were used across patients without time-lock, most of these clinically significant effects observed in our study would likely be averaged out. A key observation is that the sLI time courses of many clinical variables were markedly different from those of mLI and nLI patients, indicating that sLI is associated with more severe disease. For example, LDH, procalcitonin, and ferritin were significantly higher at 

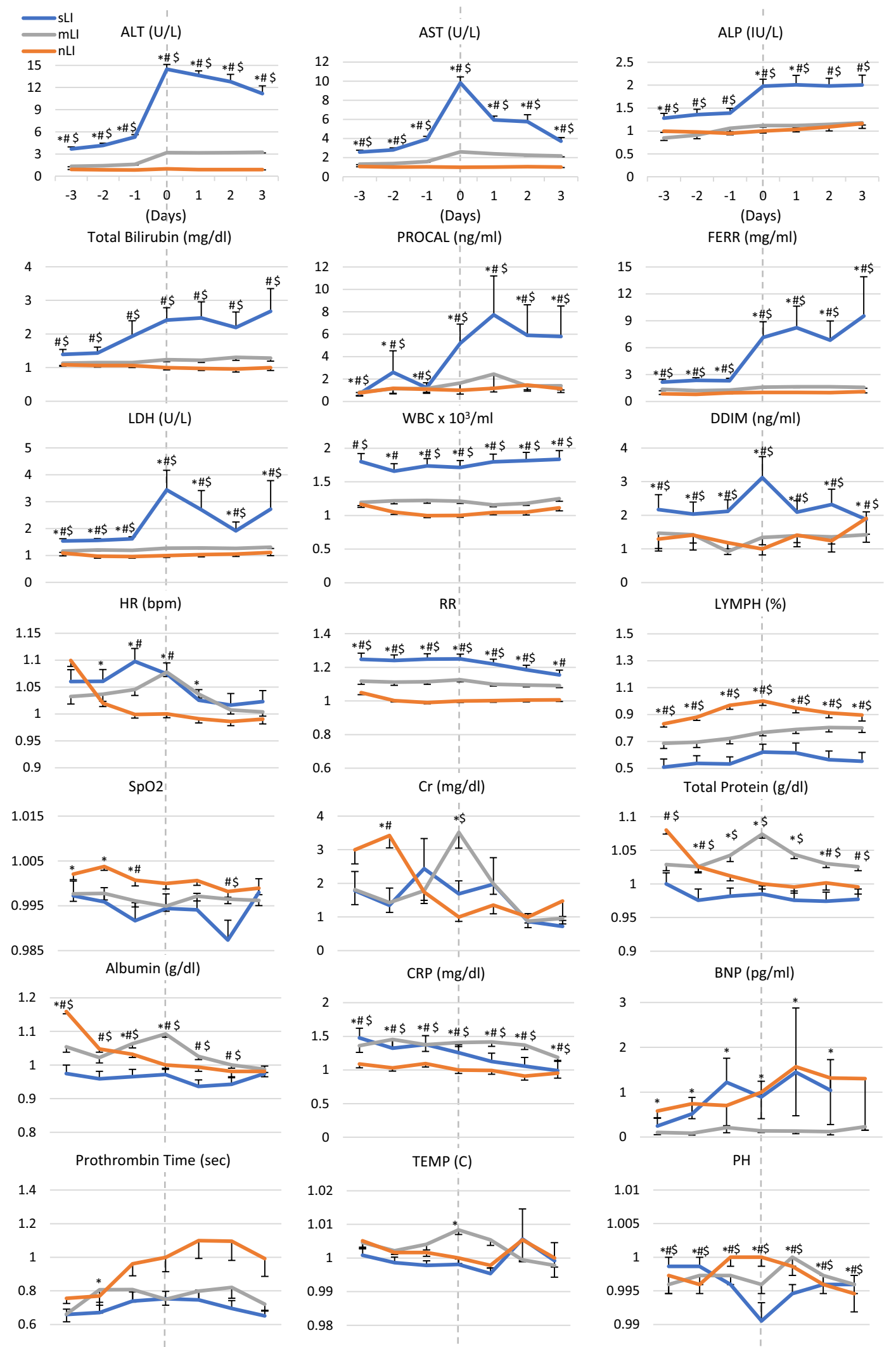
4Fig. 3 Temporal progression of laboratory tests, vitals and blood gases with time 0 representing day of liver injury onset in liver injury patients. No liver injury patient data were also centered around $3^{\text {rd }}$ day after hospital admission. Values are normalized by dividing all data points by value of reading at time 0 of no LI group. $\mathrm{Cr}$ creatinine, $B N P$ brain natriuretic peptide, $A L T$ alanine aminotransferase, $A S T$ aspartate aminotransferase, $P R O C A L$ procalcitonin, $C R P$ C-reactive protein, $L D H$ lactate dehydrogenase, $W B C$ white blood cell, no $L I$ no liver injury is the orange line. mild LI is the grey line, mild liver injury. Severe LI, severe liver injury is the blue line. Error bars are SEM. *Significant between no LI and mild LI, " Significant between no LI and severe, and \$Significant between mild LI and severe LI based on linear mixed models

Table 2 Performance metrics (AUCs) of the individual top predictors of (A) mild and severe liver injury and (B) severe injury only at different days prior to onset

\begin{tabular}{llllll}
\hline & Day-3 & Day-2 & & Day-1 & Day 0 \\
\hline (A) & & & & & \\
ALT & 0.67 & 0.75 & & 0.83 & 0.93 \\
AST & 0.61 & 0.66 & & 0.71 & 0.88 \\
LDH & 0.5 & 0.63 & & 0.69 & 0.61 \\
LYMPH & 0.5 & 0.61 & & 0.64 & 0.51 \\
RR & 0.52 & 0.62 & & 0.69 & 0.5 \\
Combined & 0.68 & 0.78 & & 0.79 & 0.81 \\
(B) & & & & & \\
ALT & 0.84 & & 0.86 & 0.9 & 0.98 \\
AST & 0.71 & & 0.75 & 0.85 & 0.95 \\
LDH & 0.5 & & 0.62 & 0.61 & 0.8 \\
RR & 0.5 & & 0.61 & 0.57 & 0.7 \\
FERR & 0.55 & & 0.53 & 0.52 & 0.69 \\
LYMPH & 0.5 & & 0.5 & 0.5 & 0.64 \\
Combined & 0.84 & & 0.88 & 0.92 & 0.98 \\
\hline
\end{tabular}

For combined models using all top variables shown, AUCs determination excluded AST due to its strong correlation with ALT

the time of sLI onset as compared to the onset of $\mathrm{mLI}$ and $\mathrm{nLI}$, and these elevations suggest more severe systemic inflammation in sLI. Similarly, lymphocyte count was significantly lower in $\mathrm{SLI}$ compared to $\mathrm{mLI}$ and $\mathrm{nLI}$, indicative of greater immunological stress in sLI [27]. It is not surprising that the time courses of AST, ALP, and total bilirubin were similar to that of ALT given that AST, ALP, and total bilirubin can reflect both hepatocellular and cholestatic injury [23, 24]. The indicators that change early on and gradually worsen can serve as early warning signs of liver injury because they allow physicians to intervene and, thus, should be closely monitored in COVID-19 patients.

Although AST, ALT, ALP, and total bilirubin are commonly measured to assess liver functions and their elevations have been associated with increased mortality risk in COVID-19 [22], we chose to time lock to ALT because ALT is more liver specific than AST [23-25]. AST may also be elevated by acute injury to the heart, kidney, and brain, which are also affected by COVID-19 [24, 25]. We did not time lock to bilirubin or ALP because elevations in bilirubin can also indicate extrahepatic disorders and ALP can also be elevated by kidney injury [24, 25].

\section{Potential mechanisms of liver injury}

The mechanisms of liver injury associated with COVID-19 are incompletely understood. The liver could be damaged by direct viral infection, indirect host-immune responses, and treatment drug toxicity [11-13]. Direct SARS-CoV-2 infection is mediated by the angiotensin-converting enzyme 2 (ACE2) receptor [28]. The observed expression of ACE2 is far greater in cholangiocytes of the bile duct epithelium than in hepatocytes of the liver parenchyma, suggesting that direct cytopathic target for SARS-CoV-2 is cholangiocytes [29]. We found that ALP, a measure of bile duct injury, is not as severely elevated as AST and ALT during the time course of both mild and severe LI. Thus, we cannot yet conclude that COVID-19 liver injury is driven by direct tropism of cholangiocytes. Hypoxia, a relatively common complication of COVID-19, is also known to regulate hepatocyte ACE2 expression [12, 30]. Our results show that both $\mathrm{mLI}$ and sLI patients display elevation in respiratory rate as compared to nLI patients, suggesting that persistent hypoxic conditions might be upregulating ACE2 expression in hepatocytes to contribute to direct liver injury over the course of hospitalization. Secondary damage to the liver, such as by cytokine stormdriven inflammation and the resultant distributive shock, is another plausible mechanism of LI [11, 12]. Procalcitonin, ferritin, and D-dimer were elevated before the threshold for severe LI is met. Both procalcitonin and ferritin are markers of inflammation, LDH is a marker of cell death across different organs, and D-dimer is an indicator of blood clots associated with early mortality in COVID-19 [27]. The increasing elevation of these markers prior to the onset of sLI is suggestive of inflammation and vasculopathy at the systemic level which may cause organ injury [31]. Note that procalcitonin, ferritin, and LDH are not only associated with systemic inflammation, but are also associated with bacterial infections, and therefore their observed elevations may be the result of bacterial co-infection or secondary infection in COVID-19 [32]. In particular, procalcitonin is known to be highly correlated with bacterial infection [33]. However, concomitant bacterial infection has been shown to be relatively infrequent in COVID-19, occurring in an estimated $8.1 \%$ of critically ill patients [34]. In this study, we did not collect data on specific COVID-19-related infections and further studies 
are needed to evaluate different COVID-19-related infections and treatments.

Taken together, given the relative late onset of sLI, high rate of IMV, anticoagulant and steroid use in SLI, there was likely hepatotoxicity from COVID-19 therapies that include antiviral, antibacterial, steroids, anticoagulants, and immuno-modulatory drugs, amongst others. In particular, the disproportional host-immune responses (including inflammation) are known to cause liver injury in COVID-19 patients $[11-14,35]$. Alternative interpretations are possible and prospective studies are warranted.

\section{Predictive model using a few clinical variables accurately predicts liver injury a few days prior}

The top predictors of both sLI and mLI versus sLI only were essentially identical and they were ALT, AST, LDH, $\mathrm{RR}$, ferritin and lymphocyte, which is encouraging. Prediction performance of sLI was overall better than that of both sLI and $\mathrm{mLI}$, as expected, because the clinical variables of $\mathrm{mLI}$ were similar to those of $\mathrm{nLI}$, but both were different from those of sLI. We were able to construct a prediction model using these longitudinal clinical variables to predict liver injury. The predictive model using these combined predictors yielded excellent prediction performance with a $0.84-0.98$ AUC at $0-3$ days prior to sLI. This is the first time that temporal progression of clinical variables is used to predict COVID-19-related liver injury at different days prior to onset. Additional testing of this predictive model using independent dataset from different hospitals is warranted.

\section{Comparison with AKI suggests that pathogenesis of liver injury and AKI is likely different}

It is informative to compare in-hospital liver injury with inhospital AKI in the same cohorts of COVID-19 patients [18], as AKI is also known to be associated with higher mortality rate. Overall, sLI prevalence was $9.18 \%$ and mLI prevalence was $39.38 \%$, compared to AKI prevalence of $29.9 \%$. In patients with ICU care, sLI prevalence was $23.2 \%$ and mLI prevalence was $46.7 \%$ had $\mathrm{mLI}$, compared to AKI prevalence of $37.3 \%$. sLI had a higher IMV rate than AKI ( $52 \%$ vs $35.3 \%$ ), higher steroid use (52.0\% vs $36.2 \%)$, and higher dialysis rate (16\%, vs $1.7-8.5 \%)$ but similar anticoagulant use $(58.4 \%$ vs $53.9 \%$ for prophylactic, and $33.6 \%$ vs $28.7 \%$ for therapeutic AC). Adjusted odds ratio of sLI (OR $=1.37$ [95\% CI 1.10, 1.70] $p=0.005)$ was lower than that of AKI (OR $=4.67$ [95\% CI 3.1, $7.0], p<0.001$ vs non-AKI). sLI onset was 9.4 days whereas the AKI onset was 3.3 days. Of note, $69.6 \%$ of patients had both sLI and AKI, $49.8 \%$ of patients had both mLI and AKI, and $35.6 \%$ nLI patients had no AKI. These comparisons suggest that the pathogenesis of liver injury and AKI is likely different, although there could be some common elements.

\section{Limitations}

There are several limitations in this study. First, although the majority of patient records did not indicate pre COVID19 liver disease, undiagnosed prior history of liver injury or disease might exist. COVID-19 could make pre-existing liver disease worse. Second, this is retrospective study from a single academic hospital. These findings need to be replicated in a large retrospective cohort from other institutions as well as in form of prospective studies. Third, our study was not adequately powered to address potential ethnicity or racial differences because the majority of our cohorts were Caucasian or unknown race. Finally, it is unclear whether such liver injury is transient or persistent, although it would not be surprising that some COVID-19 survivors will be more susceptible to COVID-19-related liver complications in the future.

\section{Conclusions}

This study characterized the temporal progression of readily available clinical and laboratory variables associated with inhospital liver injury in COVID-19 patients, providing important insights in graded liver injury pathogenesis. Severe, but not mild, liver injury confers a higher risk of mortality and is an independent risk factor of worse COVID-19 outcomes. The relatively late onset of severe liver injury and more aggressive treatments suggest hepatotoxicity may in part be due to drugs used to treat COVID-19. The earliest clinical predictors of liver injury are identified, and they accurately predict severe liver injury a few days prior to onset. This approach may prove useful for better management of COVID-19 patients with liver injury.

\section{Acknowledgements None.}

Author contributions JL: collected, curated, analyzed and edited paper; HA: curated, drafted and edited paper; SF: edited and revised paper; WH: analyzed and edited paper; TD: designed, supervised, wrote paper, and approved final version.

Funding None.

Data availability The datasets used and/or analyzed during the current study are available from the corresponding author on reasonable request.

\section{Declarations}

Conflict of interest Authors declare no conflicts of interest.

Ethical approval and consent to participate This retrospective singlecenter study was approved by the Institutional Research Board of Stony brook University (IRB 2020-00207) with an exemption for informed consent and a HIPAA waiver. 


\section{References}

1. Zhu N, Zhang D, Wang W, Li X, Yang B, Song J, et al. A Novel Coronavirus from patients with pneumonia in China, 2019. N Engl J Med. 2020;382(8):727-33.

2. Huang C, Wang Y, Li X, Ren L, Zhao J, Hu Y, et al. Clinical features of patients infected with 2019 novel coronavirus in Wuhan. China Lancet. 2020;395(10223):497-506.

3. Wu J, Song S, Cao HC, Li LJ. Liver diseases in COVID-19: Etiology, treatment and prognosis. World J Gastroenterol. 2020;26(19):2286-93.

4. Del Zompo F, De Siena M, Ianiro G, Gasbarrini A, Pompili M, Ponziani F. Prevalence of liver injury and correlation with clinical outcomes in patients with COVID-19: systematic review with meta-analysis. Eur Rev Med Pharmacol Sci. 2020;24(24):13072-88.

5. Amiri-Dashatan N, Koushki M, Ghorbani F, Naderi N. Increased inflammatory markers correlate with liver damage and predict severe COVID-19: a systematic review and meta-analysis. Gastroenterol Hepatol Bed Bench. 2020;13(4):282.

6. Medetalibeyoglu A, Catma Y, Senkal N, Ormeci A, Cavus B, Kose $\mathrm{M}$, et al. The effect of liver test abnormalities on the prognosis of COVID-19. Ann Hepatol. 2020;19(6):614-21.

7. Marjot T, Webb GJ, Barritt AST, Moon AM, Stamataki Z, Wong VW, et al. COVID-19 and liver disease: mechanistic and clinical perspectives. Nat Rev Gastroenterol Hepatol. 2021;18(5):348-64.

8. Kunutsor SK, Laukkanen JA. Markers of liver injury and clinical outcomes in COVID-19 patients: a systematic review and metaanalysis. J Infect. 2021;82(1):159-98.

9. Feng G, Zheng KI, Yan QQ, Rios RS, Targher G, Byrne CD, et al. COVID-19 and liver dysfunction: current insights and emergent therapeutic strategies. J Clin Transl Hepatol. 2020;8(1):18-24.

10. Bangash MN, Patel J, Parekh D. COVID-19 and the liver: little cause for concern. Lancet Gastroenterol Hepatol. 2020;5(6):529-30.

11. Brito CA, Barros FM, Lopes EP. Mechanisms and consequences of COVID-19 associated liver injury: What can we affirm? World J Hepatol. 2020;12(8):413-22.

12. Nardo AD, Schneeweiss-Gleixner M, Bakail M, Dixon ED, Lax SF, Trauner M. Pathophysiological mechanisms of liver injury in COVID-19. Liver Int. 2021;41(1):20-32.

13. Syed A, Khan A, Gosai F, Asif A, Dhillon S. Gastrointestinal pathophysiology of SARS-CoV2-a literature review. J Commun Hospital Intern Med Perspect. 2020;10(6):523-8.

14. Han M-W, Wang M, Xu M-Y, Qi W-P, Wang P, Xi D. Clinical features and potential mechanism of coronavirus disease 2019-associated liver injury. World J Clin Cases. 2021;9(3):528.

15. Vandenbroucke JP, von Elm E, Altman DG, Gøtzsche PC, Mulrow $\mathrm{CD}$, Pocock SJ, et al. Strengthening the reporting of observational studies in epidemiology (STROBE): explanation and elaboration. PLoS Med. 2007;4(10):e297.

16. Zhao Z, Chen A, Hou W, Graham JM, Li H, Richman PS, et al. Prediction model and risk scores of ICU admission and mortality in COVID-19. PLoS ONE. 2020;15(7):e0236618.

17. Musheyev B, Borg L, Janowicz R, Matarlo M, Boyle H, Singh $\mathrm{G}$, et al. Functional status of mechanically ventilated COVID19 survivors at ICU and hospital discharge. J Intensive Care. 2021;9(1):31.

18 Lu J, Babatsikos I, Fisher MC, Hou W, Duong TQ. Longitudinal clinical profiles of community- versus hospital-acquired acute kidney injury in COVID-19 patients. Front Med. 2021;8:587.

19. Lam KW, Chow KW, Vo J, Hou W, Li H, Richman PS, et al. Continued in-hospital ACE inhibitor and ARB Use in hypertensive
COVID-19 patients is associated with positive clinical outcomes. J Infect Dis. 2020;222(8):1256-64.

20. Cohen JP, Dao L, Roth K, Morrison P, Bengio Y, Abbasi AF, et al. Predicting COVID-19 pneumonia severity on chest X-ray with deep learning. Cureus. 2020;12(7):e9448.

21 Frager SZ, Szymanski J, Schwartz JM, Massoumi HS, Kinkhabwala M, Wolkoff AW. Hepatic predictors of mortality in severe acute respiratory syndrome Coronavirus 2: role of initial aspartate aminotransferase/alanine aminotransferase and preexisting cirrhosis. Hepatol Commun. 2020;5:424.

22. Lei F, Liu YM, Zhou F, Qin JJ, Zhang P, Zhu L, et al. Longitudinal association between markers of liver injury and mortality in COVID-19 in China. Hepatology. 2020;72(2):389-98.

23 Kwo PY, Cohen SM, Lim JK. ACG clinical guideline: evaluation of abnormal liver chemistries. Off J Am Coll Gastroenterol ACG. 2017;112(1):18-35.

24. Green RM, Flamm S. AGA technical review on the evaluation of liver chemistry tests. Gastroenterology. 2002;123(4):1367-84.

25. Giannini EG, Testa R, Savarino V. Liver enzyme alteration: a guide for clinicians. CMAJ. 2005;172(3):367-79.

26. Yadlapati S, Lo KB, DeJoy R, Gul F, Peterson E, Bhargav R, et al. Prevailing patterns of liver enzymes in patients with COVID-19 infection and association with clinical outcomes. Ann Gastroenterol. 2021;34(2):224-8.

27 Tjendra Y, Al Mana AF, Espejo AP, Akgun Y, Millan NC, GomezFernandez $\mathrm{C}$, et al. Predicting disease severity and outcome in COVID-19 patients: a review of multiple biomarkers. Arch Pathol Lab Med. 2020;144(12):1465-74.

28 Zhang H, Penninger JM, Li Y, Zhong N, Slutsky AS. Angiotensinconverting enzyme 2 (ACE2) as a SARS-CoV-2 receptor: molecular mechanisms and potential therapeutic target. Intensive Care Med. 2020;46(4):586-90.

29. Kleiner DE. Liver biopsy shines a light on COVID-19-related liver injury. Cell Mol Gastroenterol Hepatol. 2021;11(3):881-2.

30. Paizis G, Tikellis C, Cooper ME, Schembri JM, Lew RA, Smith AI, et al. Chronic liver injury in rats and humans upregulates the novel enzyme angiotensin converting enzyme 2 . Gut. 2005;54(12):1790-6.

31 Becker RC. COVID-19-associated vasculitis and vasculopathy. New York: Springer; 2020.

32. Suárez-Santamaría M, Santolaria F, Pérez-Ramírez A, AlemánValls M-R, Martínez-Riera A, González-Reimers E, et al. Prognostic value of inflammatory markers (notably cytokines and procalcitonin), nutritional assessment, and organ function in patients with sepsis. Eur Cytokine Netw. 2010;21(1):19-26.

33. Simon L, Gauvin F, Amre DK, Saint-Louis P, Lacroix J. Serum procalcitonin and $\mathrm{C}$-reactive protein levels as markers of bacterial infection: a systematic review and meta-analysis. Clin Infect Dis. 2004;39(2):206-17.

34. Langford BJ, So M, Raybardhan S, Leung V, Westwood D, MacFadden DR, et al. Bacterial co-infection and secondary infection in patients with COVID-19: a living rapid review and meta-analysis. Clin Microbiol Infect. 2020;26:1622.

35. Ommati MM, Mobasheri A, Heidari R. Drug-induced organ injury in coronavirus disease 2019 pharmacotherapy: mechanisms and challenges in differential diagnosis and potential protective strategies. J Biochem Mol Toxicol. 2021;11:e22795.

Publisher's Note Springer Nature remains neutral with regard to jurisdictional claims in published maps and institutional affiliations. 